\title{
The PI3K/Akt inhibitor LY294002 reverses BCRP-mediated drug resistance without affecting BCRP translocation
}

\author{
YASUO IMAI $^{1}$, MAYUMI YOSHIMORI ${ }^{1}$, KAZUNORI FUKUDA $^{2}$, \\ HIDETSUGU YAMAGISHI $^{1}$ and YOSHIHIKO UEDA ${ }^{1}$ \\ ${ }^{1}$ Department of Pathology and ${ }^{2}$ Joint Research Center, Dokkyo Medical University \\ Koshigaya Hospital, Koshigaya, Saitama 343-8555, Japan \\ Received December 14, 2011; Accepted February 23, 2012
}

DOI: $10.3892 /$ or.2012.1724

\begin{abstract}
Cellular responses toward cytotoxic drugs are influenced by crosstalk between oncogenic signals and resistance mechanisms. Inhibition of the PI3K/Akt pathway is effective in sensitizing cancer cells of various organs, although the mechanisms largely remain to be elucidated. Breast cancer resistance protein (BCRP)/ABCG2, a drug efflux pump, confers resistance to multiple anticancer agents such as SN-38 and topotecan. Previous studies reported that inhibition of the PI3K/Akt pathway, by gene knockout or PI3K inhibitors, modulated BCRP-mediated drug transport via BCRP translocation in hematopoietic stem cells, renal polarized cells and glioma stem-like cells of mammals. In this study, we assessed the effects of PI3K inhibitors, LY294002 and wortmannin, on BCRP-mediated anticancer drug resistance of human cancer MCF-7 and A431 cells. LY294002, but not wortmannin, reversed the BCRP-mediated SN-38 and topotecan resistance. LY294002 treatment did not affect total or cell surface BCRP levels as determined by western blotting and flow cytometry but blocked BCRP-mediated topotecan efflux in a dose-dependent manner. Immunohistochemical analyses also demonstrated unchanged cellular BCRP distribution. BCRP overexpression in MCF-7 and A431 cells did not confer LY294002 resistance, suggesting that LY294002 is not a transported substrate of BCRP. LY294002 is a derivative of quercetin, a member of flavonoids. Taken together, these results suggest that LY294002 inhibits BCRP-mediated drug transport not by BCRP translocation through the PI3K/ Akt signal but putatively as a competitive inhibitor in a major subset of cancer cells. Due to its dual effects, LY294002 could be a lead compound for developing more effective and tolerable reagents for cancer treatment.
\end{abstract}

Correspondence to: Dr Yasuo Imai, Department of Pathology, Dokkyo Medical University, Koshigaya Hospital, 2-1-50 MinamiKoshigaya, Koshigaya, Saitama 343-8555, Japan

E-mail: ya-imai@dokkyomed.ac.jp

Key words: breast cancer resistance protein, LY294002, PI3K/Akt, drug resistance

\section{Introduction}

The outcome of cancer chemotherapy is affected by multifactorial resistance mechanisms. One of the well-studied effectors of drug resistance are the membrane ATP-binding cassette (ABC) transporters, particularly P-glycoprotein/ MDR1, the multidrug resistance related proteins MRP1, and breast cancer resistance protein (BCRP)/ABCG2 (1). Other mechanisms of drug resistance involve alteration in the apoptotic response, activation of DNA repair or stimulation of detoxifying systems, such as the exocytotic pathway for reducing intracellular drug concentration $(2,3)$. On the other hand, cellular responses toward cytotoxic drugs are also controlled by crosstalk between oncogenic signaling pathways and resistance mechanisms. For example, enhanced sensitivity to drug treatment was observed in the presence of signaling inhibitors. Phosphatidylinositol 3-kinase (PI3K) inhibition was particularly effective in sensitizing pancreatic adenocarcinoma cells (4), ovarian cancer cells $(5,6)$, and breast cancer cells $(7,8)$. However, the mechanisms of how PI3K/Akt signaling pathways control drug-mediated apoptosis as well as resistance to drug-induced cell death are poorly understood.

BCRP, a half-size ABC transporter (9-11), localizes in the apical membrane of cells, across which its substrates are excreted out of cells. BCRP transports certain chlorophyll metabolites, steroid metabolites and xenobiotics and is presumed to play a protective role against toxic substances in the maternal-placental barrier, the digestive tract and the blood-testis barrier (12-15). BCRP is a molecular determinant of side-population (SP) cells, which are enriched in various stem cells (16). It also mediates the concurrent resistance to chemotherapeutic agents, such as mitoxantrone, SN-38 (an active metabolite of irinotecan), topotecan and gefitinib, by pumping them out of the cell $(9-11,17-19)$.

Previous studies reported that inhibition of the PI3K/Akt pathway, by gene knockout or PI3K inhibitors, modulated BCRP-mediated drug transport via BCRP translocation in SP cells in the bone marrow, renal polarized cells, and glioma stem-like cells of mammals (20-22). In the present study, we examined the effects of two representative PI3K inhibitors, LY294002 and wortmannin, on the BCRP-mediated drug resistance. We show that LY294002 reversed BCRP-mediated drug resistance in human cancer cells but wortmannin did not. 
LY294002 treatment affected neither BCRP expression levels nor subcellular localization. LY294002 inhibited BCRPmediated drug efflux in a dose-dependent manner, but BCRP overexpression in cancer cells did not result in LY294002 resistance. Our results hence suggest that LY294002 reverses BCRP-mediated drug resistance without BCRP translocation and may function as a competitive inhibitor of BCRP-mediated transport in a major subset of cancer cells.

\section{Materials and methods}

Reagents. LY294002, wortmannin and estrone were purchased from Wako (Osaka, Japan). Topotecan was purchased from Bosche Scientific (New Brunswick, NJ, USA) and gefitinib was obtained from Toronto Research Chemicals (North York, Ontario, Canada). SN-38 was generously provided by Yakult Honsha (Tokyo, Japan).

Establishment of BCRP-transfected cells. Breast cancer MCF-7 cells and epidermoid carcinoma A431 cells were maintained in high-glucose Dulbecco's modified Eagle's medium (DMEM) supplemented with 7\% fetal bovine serum (FBS) at $37^{\circ} \mathrm{C}$ in a humidified incubator with $5 \% \mathrm{CO}_{2}$. Establishment of MCF-7/BCRP cells has been previously described (23). A BCRP cDNA fragment was inserted into the pCAGIPuro plasmid (pCAGIPuro-BCRP) (24). A431 cells that stably expressed exogenous BCRP (A431/BCRP) were established by lipofection with pCAGIPuro-BCRP. The transfected cells were selected with $0.5 \mu \mathrm{g} / \mathrm{ml}$ puromycin for 7 days, and $>100$ clones of resistant cells were mixed and pooled for subsequent experiments. Expression levels of exogenous BCRP were stable for at least six months.

Cell growth inhibition assay. Effects of PI3K inhibitors on cell growth were evaluated by measuring cell growth after incubation at $37^{\circ} \mathrm{C}$ for 4 days in the absence or presence of various concentrations of each PI3K inhibitor. Cells were seeded in 12-well plates in the absence or presence of each PI3K inhibitor for 4 days. Cell numbers were determined by using a Coulter counter (Beckman Coulter, Fullerton, CA, USA) and presented as percentages relative to those of control cells.

Next, effects of the PI3K inhibitors on anticancer drug resistance were evaluated by measuring cell growth after incubation at $37^{\circ} \mathrm{C}$ for 4 days in the absence or presence of various concentrations of anticancer drugs in combination with each PI3K inhibitor. Cell numbers were determined and presented as percentages relative to those of control cells cultured in the absence of anticancer drugs.

Western blot analysis. Cells $\left(1 \times 10^{6}\right)$ were incubated in DMEM with or without various concentrations of LY294002 for $24 \mathrm{~h}$. Western blot analysis was performed under the reducing conditions as previously described (23). The blots were incubated with $5 \mu \mathrm{g} / \mathrm{ml}$ of the mouse anti-BCRP monoclonal antibody, BXP-21 (Chemicon, Temecula, CA, USA). GAPDH expression was also analyzed as a loading control.

Fluorescence-activated cell sorting (FACS) analyses. Effects of specific compounds on the cellular accumulation of topotecan were determined by flow cytometry. Trypsinized cells

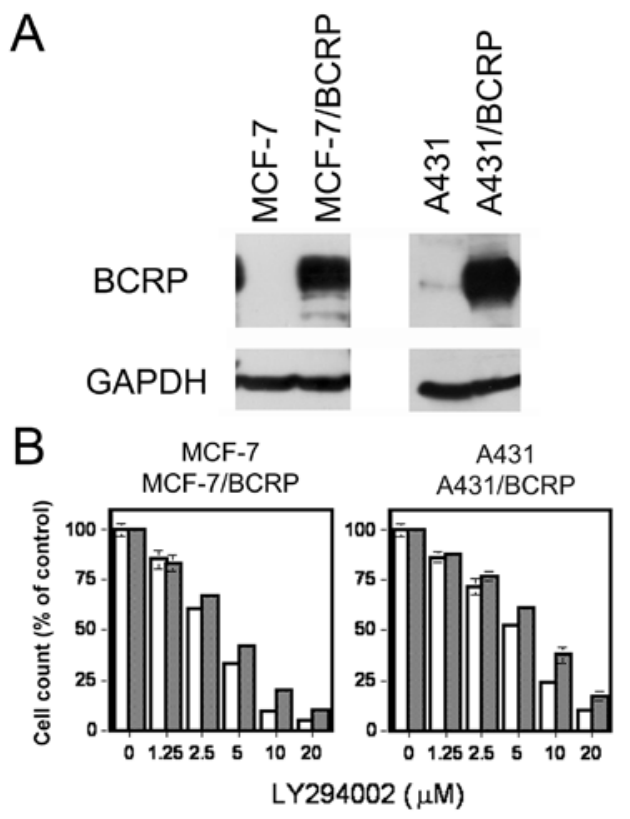

Figure 1. (A) BCRP expression in the $B C R P$-transfected cells. Protein sample (20 $\mu \mathrm{g}$ of MCF-7 and MCF-7/BCRP and $10 \mu \mathrm{g}$ of A431 and A431/BCRP) was loaded in each lane, and western blot analysis was performed under the reducing conditions. BCRP was detected using BXP-21. (B) LY294002 resistance of the $B C R P$-transfected cells. MCF-7/BCRP and MCF-7 cells $\left(3 \times 10^{4} /\right.$ well) as well as A431/BCRP and A431 cells (5x103/well) were seeded into 12 -well plates, and cultured in the absence or presence of various concentrations of LY294002 for 4 days. Cell numbers were determined, and presented as percentages relative to those of control cells cultured without LY294002. Left, MCF-7 and MCF-7/BCRP cells. Right, A431 and A431/BCRP cells. Open bars, parental cells. Dotted bars, $B C R P$-transfected cells. The data shown are the means \pm SD of triplicate determinations, and are representative of two independent experiments. Where a vertical bar is not shown, the SD is within the bar.

$\left(5 \times 10^{5}\right)$ were incubated in the presence of indicated concentrations of reagents for $60 \mathrm{~min}$ at $37^{\circ} \mathrm{C}$. After addition of topotecan to the medium, the cells were incubated for an additional $30 \mathrm{~min}$ at $37^{\circ} \mathrm{C}$, washed in ice-cold phosphate-buffered saline (PBS), and subjected to fluorescence analysis by using a FACSCalibur (Becton-Dickinson, San Jose, CA, USA).

Amounts of cell surface BCRP were measured by FACS. Cells were incubated in DMEM for $12 \mathrm{~h}$ without or with $2 \mu \mathrm{M}$ (MCF-7/BCRP and MCF-7) or $1 \mu \mathrm{M}$ (A431/BCRP or A431) LY294002. Trypsinized cells $\left(5 \times 10^{5}-1 \times 10^{6}\right)$ were incubated with $10 \mu \mathrm{g} / \mathrm{ml}$ of biotinylated anti-human ABCG2, 5D3 (eBiosciences, San Diego, CA, USA) or mouse IgG-BIOT conjugate (Beckman Coulter) for 30 min on ice in the dark, washed and incubated with $10 \mu \mathrm{g} / \mathrm{ml}$ of R-phycoerythrinconjugated streptavidin (Becton-Dickinson) for an additional $30 \mathrm{~min}$ on ice in the dark. After washing, the fluorescence staining level was analyzed with a FACSCalibur.

Immunofluorescence analysis of BCRP. Cells were treated with $1 \mu \mathrm{M}$ (A431/BCRP) or $2 \mu \mathrm{M}$ (MCF-7/BCRP) of LY294002 for $12 \mathrm{~h}$ and collected with cell scrapers. The cells were routinely formalin-fixed, paraffin-embedded as a cell block and processed. Antigen-retrieval was performed by autoclaving $\left(121^{\circ} \mathrm{C}\right)$ for $15 \mathrm{~min}$. The slides were then incubated with $12.5 \mu \mathrm{g} / \mathrm{ml}$ of BXP-21 at $4^{\circ} \mathrm{C}$ overnight followed by incubation with a polyclonal anti-mouse immunoglobulin/FITC 

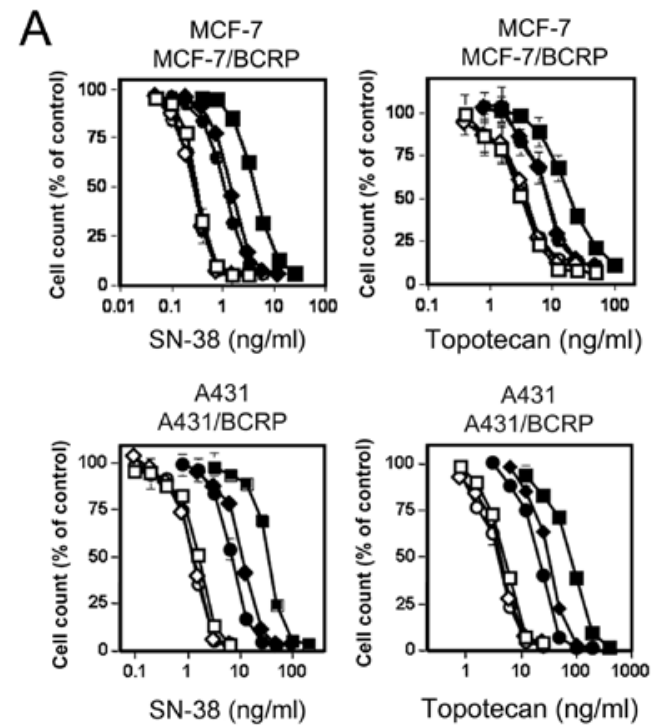

\section{B}
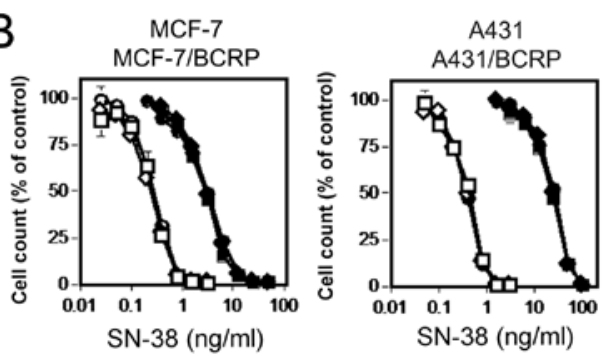

Figure 2. (A) Effects of LY294002 on anticancer drug resistances of BCRPtransfected cells. MCF-7/BCRP as well as MCF-7 cells (3x10 $/$ well) and A431/BCRP as well as A431 cells $\left(5 \times 10^{3} /\right.$ well) were seeded into 12 -well plates, and cultured in the absence or presence of various concentrations of anticancer drugs with or without LY294002 for 4 days. Cell numbers were determined, and presented as percentages relative to those of control cells cultured in the absence of anticancer agents. Open symbols, the parental cells. Closed symbols, the $B C R P$-transfected cells. The data shown are the means \pm $\mathrm{SD}$ of triplicate determinations, and are representative of three independent experiments. Where a vertical bar is not shown, the SD is within the symbol Squares, without LY294002. Diamonds, $1 \mu \mathrm{M}$ (MCF-7) or $0.5 \mu \mathrm{M}$ (A431) of LY294002. Circles, $2 \mu \mathrm{M}$ (MCF-7) or $1 \mu \mathrm{M}$ (A431) of LY294002. (B) Effects of wortmannin on SN-38 resistance of $B C R P$-transfected cells. Experimental procedures and presentation are the same as described above. Open symbols, parental cells. Closed symbols, $B C R P$-transfected cells. Squares, without wortmannin. Diamonds, $1 \mu \mathrm{M}$ (MCF-7) or $0.5 \mu \mathrm{M}$ (A431) of wortmannin. Circle, $2 \mu \mathrm{M}$ (MCF-7) or $1 \mu \mathrm{M}$ (A431) of wortmannin.

secondary antibody (1:20 dilution) (Dako, Glostrup, Denmark) at $4^{\circ} \mathrm{C}$ for $2 \mathrm{~h}$. BCRP was detected by fluorescence microscopy as green fluorescence and cell nuclei were indicated by purple fluorescence.

\section{Results}

Effects of LY294002 and wortmannin on drug resistances of BCRP-transfected cells. MCF-7/BCRP and A431/BCRP cells were found to overexpress BCRP (Fig. 1A) and they showed significantly higher resistances to SN-38 and topotecan than the corresponding parental cells. These transfected cells also acquired slightly more resistance to the PI3K inhibitors (Fig. 1B and data not shown), but the degrees of resistance did not seem to be significant considering the degrees of resistance against anticancer drugs. LY294002 at concentrations $<2 \mu \mathrm{M}$

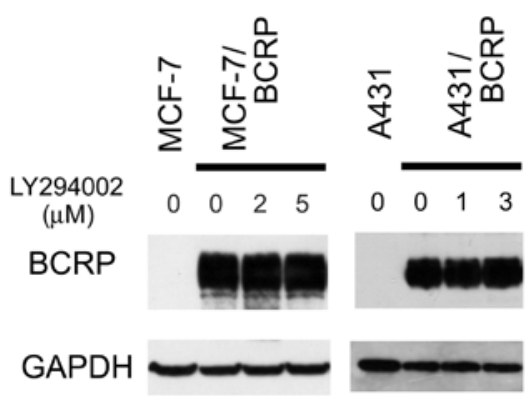

Figure 3. Effects of LY294002 on BCRP protein expression. Cells were cultured in the absence or presence of various concentrations of LY294002 for $24 \mathrm{~h}$. After harvesting the cells, protein sample ( $20 \mu \mathrm{g}$ of MCF-7/BCRP and $5 \mu \mathrm{g}$ of A431/BCRP) was loaded in each lane and western blot analysis was performed. The data are representative of three independent experiments.

showed growth inhibitory effects $<30 \%$ on MCF-7 and A431 cells after 4-day treatment. LY294002 significantly enhanced the cytotoxicities of SN-38 and topotecan on MCF-7/BCRP and A431/BCRP cells in a dose-dependent manner, while it only slightly affected the cytotoxicities on MCF-7 and A431 cells (Fig. 2A). Wortmannin at the concentrations of 2 (for MCF-7 cells) or $1 \mu \mathrm{M}$ (for A431 cells) inhibited cell growth approximately by $30 \%$ after 4 -day treatment (data not shown) but did not show any significant reversing effects of BCRPmediated drug resistances in these cells (Fig. 2B).

Effects of LY294002 on BCRP expression. Since LY294002 treatment reversed BCRP-mediated multidrug resistance, BCRP expression levels in the transfectants were investigated by western blot analysis. Total BCRP levels in MCF-7/BCRP and A431/BCRP cells were not affected by the treatment with up to 5 or $3 \mu \mathrm{M}$ of LY294002, respectively (Fig. 3).

Effects of LY294002 on cellular topotecan uptake in BCRPtransfected cells. Next, effects of LY294002 on cellular accumulation of topotecan were evaluated by flow cytometric analysis. Cellular topotecan uptake increased in the parental MCF-7 and A431 cells in a dose-dependent manner, but not in the BCRP-transfected cells, in the absence of the reagents (Figs. 4 and 5). Treatment with LY294002 increased cellular topotecan uptake in MCF-7/BCRP in a dose-dependent manner (Fig. 4). The effects were somewhat weaker than those of gefitinib but were stronger than estrone, both known to be BCRP inhibitors $(19,25)$. Treatment of A431/BCRP cells with $10 \mu \mathrm{M}$ LY294002 in the culture medium involving $10 \mu \mathrm{M}$ topotecan revealed only marginal increase in cellular topotecan uptake, to the degree similar to estrone (Fig. 5A). However, LY294002 treatment at the concentrations of 10-20 $\mu \mathrm{M}$ clearly demonstrated increased cellular topotecan uptake in a dose-dependent manner in the medium containing $20 \mu \mathrm{M}$ of topotecan (Fig. 5B). The effects were weaker than those of gefitinib but were stronger than estrone similarly to our observations in MCF-7/BCRP cells.

FACS analysis of cell surface BCRP expression. Based on previous reports, we expected to observe BCRP translocation in the $B C R P$-transfected cells after the initiation of 


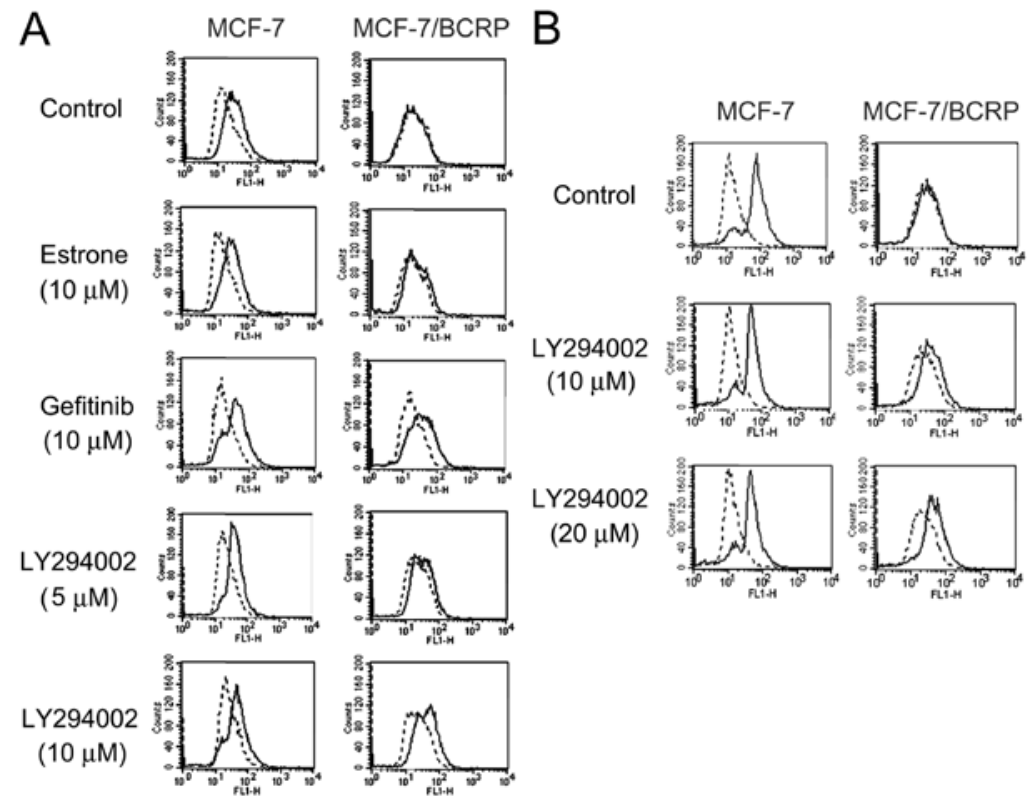

Figure 4. Effects of LY294002 on cellular topotecan uptake of MCF-7/BCRP cells. Trypsinized cells $\left(5 \times 10^{5}\right)$ were pre-incubated with indicated concentrations of reagents for $60 \mathrm{~min}$. The cells were then incubated with (solid line) or without (dotted line) (A) $20 \mu \mathrm{M}$ or (B) $40 \mu \mathrm{M}$ of topotecan for 30 min in the presence of the reagents. Finally, cellular topotecan uptake was measured by flow cytometry. The data are representative of three independent experiments.

A
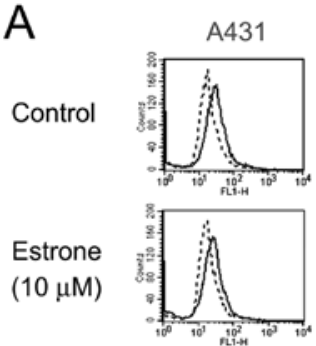

Gefitinib
$(10 \mu \mathrm{M})$

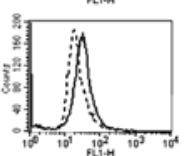

LY294002

$(5 \mu \mathrm{M})$

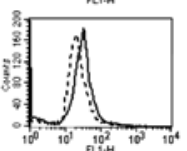

LY294002

$(10 \mu \mathrm{M})$

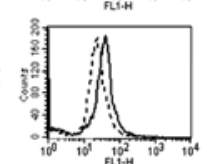

A431/BCRP
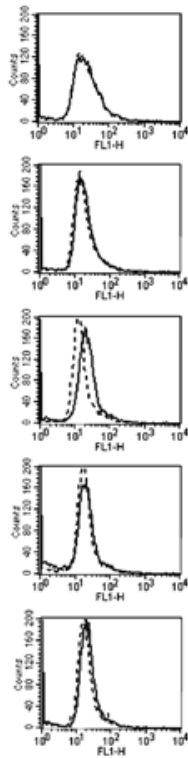

B

Control
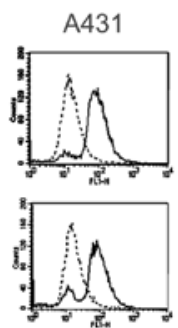

$(20 \mu \mathrm{M})$

Gefitinib

$(20 \mu \mathrm{M})$

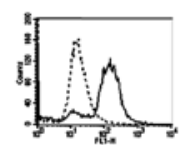

LY294002

$(10 \mu \mathrm{M})$

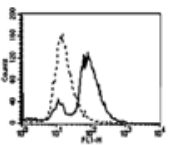

LY294002

(20 $\mu \mathrm{M})$

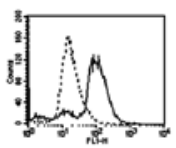

A431/BCRP
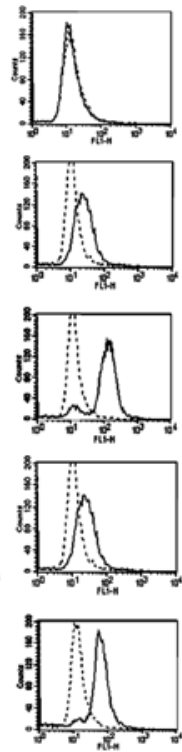

Figure 5. Effects of LY294002 on cellular topotecan uptake of A431/BCRP cells. Trypsinized cells (5x10 $)$ were pre-incubated with indicated concentrations of reagents for $60 \mathrm{~min}$. The cells were then incubated with (solid line) or without (dotted line) (A) $10 \mu \mathrm{M}$ or (B) $20 \mu \mathrm{M}$ of topotecan for $30 \mathrm{~min}$ in the presence of the reagents. Finally, cellular topotecan uptake was measured by flow cytometry. The data are representative of three independent experiments.

LY294002 treatment. BCRP localization on the cell surface after LY294002 treatment was confirmed by FACS. Although endogenous BCRP was scarcely detected by western blot analysis under the reducing conditions (Fig. 1A), considerable amounts of BCRP were detected on the cell surface of MCF-7 and A431 cells by FACS analysis (Fig. 6). It was also confirmed by FACS that BCRP-transfected cells expressed significantly greater amounts of BCRP on the cell surface than the corresponding parental cells. However, contrary to our expectation, amounts of BCRP expressed on the cell surface were similar with and without LY294002 treatment in both parental and $B C R P$-transfected cells. This surprising result suggested that trafficking problem was not a main reason for the loss of BCRP function mediated by LY294002.

Immunofluorescence analyses of the subcellular BCRP localization. We next observed BCRP expression in the cells with or without LY294002 treatment by fluorescence 


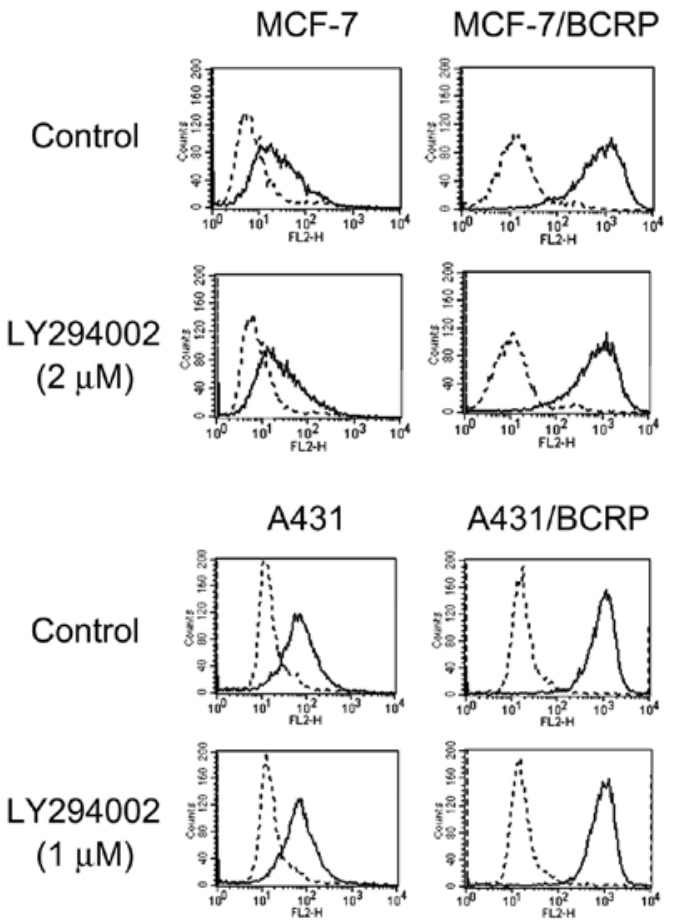

Figure 6. FACS analysis of BCRP expression on the cell surface. Cells were incubated for $12 \mathrm{~h}$ with or without $2 \mu \mathrm{M}$ (MCF-7/BCRP and MCF-7) or $1 \mu \mathrm{M}\left(\mathrm{A} 431 / \mathrm{BCRP}\right.$ or A431) LY294002. Trypsinized cells $\left(5 \times 10^{5}-10^{6}\right)$ were incubated with either biotinylated mouse IgG or anti-human ABCG2, 5D3, washed and incubated with R-phycoerythrin-conjugated streptavidin. Bold lines indicate fluorescence in the presence of 5D3 and dotted lines indicate control fluorescence in the presence of mouse IgG

immunocytochemistry. The $B C R P$-transfected cells were found to express BCRP mainly on the plasma membrane, but translocation of BCRP was not observed in the cells treated with LY294002 in this experimental setting (Fig. 7). Since LY294002 at the concentrations used in these experiments reversed BCRP-mediated drug resistance to a significant degree (Fig. 2A), these results clearly demonstrated that LY294002 can reverse BCRP-mediated drug resistance without BCRP translocation and suggest its role as a competitive inhibitor.

\section{Discussion}

For the past decade, there is growing evidence that alterations of the oncogenic signaling pathways can affect cellular responses to anticancer drugs. PI3K enzymes are regulated by growth factors and serve to phosphorylate phospholipids at the plasma membrane. Activated PI3K phosphorylates phosphatidylinositol bisphosphate $\left(\mathrm{PIP}_{2}\right)$ and generates phosphatidylinositol trisphosphate $\left(\mathrm{PIP}_{3}\right)$, which acts as a second messenger. Akt interacts with $\mathrm{PIP}_{3}$, subsequently translocates to the plasma membrane, and plays a critical role in controlling the balance between survival and apoptosis. In addition to that, PI3K/Akt inhibitors have been known to cause more favorable outcomes when co-administered with usual anticancer drugs. Several mechanisms have been postulated, such as alterations of intracellular trafficking of anticancer drugs and/or the DNA repair system, and deregulation of drug transporters. In this study, we demonstrated that the PI3K inhibitor LY294002

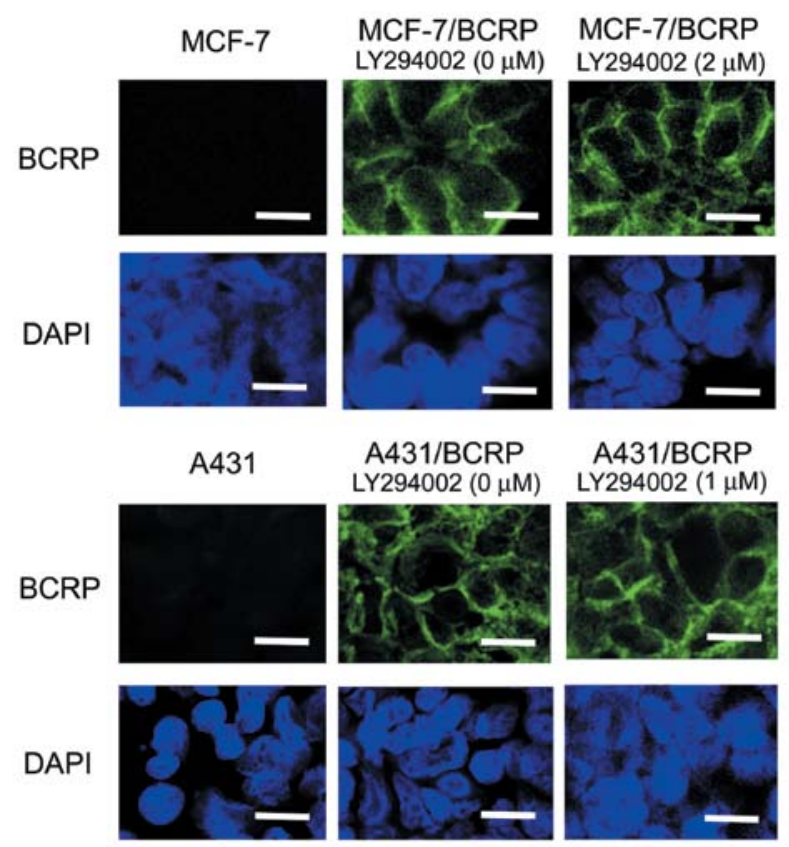

Figure 7. Immunofluorescence analyses of the subcellular BCRP localization. Cells were treated with $2 \mu \mathrm{M}$ (MCF-7/BCRP) or $1 \mu \mathrm{M}$ (A431/BCRP) LY294002 for $12 \mathrm{~h}$, collected as a cell block, formalin-fixed, paraffinembedded, and processed. BCRP is presented as green fluorescence, while cell nuclei are indicated by purple fluorescence. Scale bar, $30 \mu \mathrm{m}$.

sensitized BCRP-overexpressing cancer cells to its substrate anticancer drugs. BCRP function can be regulated through several mechanisms, including expression regulation at the RNA/protein levels and competitive block of ATP-binding or substrate transport. Previous studies reported that inhibition of the PI3K/Akt pathway caused BCRP internalization in cells. Mogi et al reported a reduced SP fraction in the bone marrow of Akt1-null mice (20). Enforced expression of Akt increased the SP fraction in the wild-type mouse bone marrow but not in the $\mathrm{Bcrp}^{-/}$bone marrow. They also showed that treatment of mouse bone marrow cells with $10 \mu \mathrm{M}$ of LY294002 for $30 \mathrm{~min}$ depleted the SP cell population. Then, after $30 \mathrm{~min}$ of incubation without LY294002, the SP cell fraction recovered to the untreated cell levels. Finally, they showed that treatment of SP cells with $10 \mu \mathrm{M}$ of LY294002 for 90 min promoted BCRP translocation from the plasma membrane to the intracellular compartment. The depletion of SP cells in the bone marrow was attributable to BCRP internalization by inhibiting the PI3K/Akt pathway.

Alteration of subcellular BCRP localization was also observed in porcine non-SP cells, LLC-PK1 (21). Treatment with $20 \mu \mathrm{M}$ of LY294002 for 90 min caused BCRP internalization from the cell surface. Transfection of the dominant-negative Akt plasmid resulted in internalization of a part of BCRP in LLC-PK1 cells. Similar results have been reported concerning glioma tumor stem-like cells (22). Treatment of SP cells from PDGF-induced, PTEN-deleted, glioma cells with $20 \mu \mathrm{M}$ of LY294002 for $16 \mathrm{~h}$ resulted in internalization of BCRP from the cell surface. Changes in BCRP localization after LY294002 treatment were also observed in SP cells from BCRPtransfected human glioma cell line U87-MG. These studies led us to hypothesize that the reversal of BCRP-mediated 
drug resistance would be attributable to alteration of cellular BCRP trafficking.

However, effects of the PI3K/Akt inhibitors on BCRP function were considerably different in BCRP-transfected MCF-7 and A431 cells. First, LY294002 reversed BCRP-mediated drug resistances, but another PI3K inhibitor, wortmannin, did not. Considering that wortmannin has stronger pharmacological activities than LY294002, reversal by LY294002 could not be due to inhibition of the PI3K/Akt signal but by specific characteristics of the compound.

Next, although LY294002 treatment resulted in inhibition of drug efflux in BCRP-transfected cells, either total cellular BCRP or cell surface BCRP levels were not changed as demonstrated by western blotting and FACS analyses. The presented data are derived from experiments using 1 or $2 \mu \mathrm{M}$ of LY294002, but similar results were obtained from experiments using LY294002 up to $10 \mu \mathrm{M}$ (data not shown). In addition, immunocytochemical analyses have clearly demonstrated no recognizable effects on cellular BCRP distribution after LY294002 treatment for $12 \mathrm{~h}$ in the both cell lines.

Since spontaneous recovery of the cell surface BCRP distribution could not be excluded during the LY294002 treatment, we established EGFP-BCRP-transfected MCF-7 and A431 cells. Those cells expressed EGFP-BCRP mainly on the cell membrane and acquired resistances to BCRP-substrate anticancer drugs, which were reversed by LY294002 (data not shown). We then performed live observation of cellular EGFPBCRP distribution of living cells in the presence of LY294002 ranging from 1 to $20 \mu \mathrm{M}$ for up to $48 \mathrm{~h}$, and could not observe alterations in the EGFP-BCRP localization at any time (data not shown). All these results suggest that LY294002 does not affect cellular BCRP distribution in the cancer cell lines.

Nevertheless, LY294002 was found to exert inhibitory effects on BCRP-mediated topotecan efflux, which were stronger than estrone but weaker than gefitinib. Thus, LY294002 demonstrated BCRP-inhibitory effects different from those observed in the previous studies. To explain the reasons for this discrepancy, we postulate two possible explanations. The first one is the difference of cell characters between stem cells and non-stem cells. Regulation of subcellular BCRP distribution by the PI3K/Akt pathway might be specific to a subset of cells such as stem/stem-like cells. It is known that the $\mathrm{PI} 3 \mathrm{~K} / \mathrm{Akt}$ signaling pathway is activated and plays a role in the maintenance of pluripotency and viability of stem cells (26). BCRP is expressed on the cell surface of pluripotent stem cells but becomes downregulated with cell maturation. On the other hand, the PI3K/Akt signals have been reported to regulate activity and trafficking of some transporters and/or receptors, such as transferrin receptor, low-density lipoprotein receptor, the amino acid transporter 4F2, integrin, and Glut1 through regulating endocytosis and recycling $(27,28)$. Therefore, the PI3K/Akt signaling pathway may be involved in mechanisms of BCRP trafficking to the cell surface and maintenance of the pluripotency of stem cells. In addition, the MEK/ERK pathway is likely to act downstream of the PI3K/Akt pathway in the embryonic stem cells (26). We previously reported that inhibition of the Ras/Raf/MEK/ERK pathway results in internalization and degradation of the BCRP protein (29). Therefore, BCRP translocation caused by inhibiting PI3K/Akt signaling may be specific to stem/stem-like cells in which the

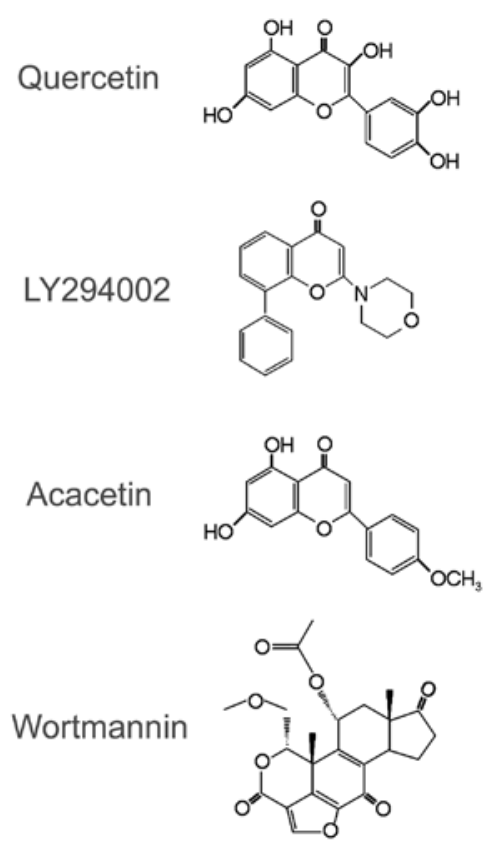

Figure 8. Chemical structures of the PI3K inhibitors and the referred flavonoids.

signal is inherently kept active. Because of a very small fraction of cancer stem cells, we might not have detected internalized BCRP in our experimental setting. Another explanation would be that some of the BCRP protein might be internalized, but expression levels of overexpessed BCRP in cancer cells are too high to discern internalized BCRP and most of BCRP would be left uninternalized. For successful internalization of the whole expressed BCRP, upregulation of proteins associated with protein trafficking might be necessary. But, we are afraid that this hypothesis cannot successfully explain the data shown in Figs. 2, 4 and 5. To explain the reversal effects, we reasoned that LY294002 would interact with the drug-binding sites of BCRP.

LY294002 was designed as a synthetic PI3K inhibitor based on quercetin, a naturally occurring flavonoid that inhibits a broad range of protein kinases $(30,31)$. Quercetin and other flavonoids have been found to reverse BCRP-mediated drug resistance to variable degrees (32). In addition, a member of the flavonoids, genistein, has been demonstrated to be subjected to BCRP-mediated transport in its native form (32). In fact, the chemical structure of LY294002 is very similar to those of quercetin and acacetin, the strongest flavonoid that reverses BCRP-mediated multidrug resistance, but the chemical structure of wortmannin, a furanosteroid metabolite of the fungus, is quite different (Fig. 8). Since BCRP overexpression did not result in acquisition of comparable degrees of the LY294002 resistance with those of anticancer drugs (Fig. 1B), we reason that LY294002 would not be a transported substrate of BCRP, but that it would competitively interact with the drug-binding site. Further investigations are needed to clarify the interaction of LY294002 with BCRP, and we are planning further experiments.

Furthermore, it has been reported that LY294002 competitively inhibited MRP1-mediated doxorubicin transport (33). Therefore, LY294002 can inhibit both MRP-1 and BCRP, 
which are two major drug transporters that cause multidrug resistance. Based on these findings, flavonoid-based PI3K inhibitors could be an attractive choice for cancer chemotherapy due to their multifunction in inhibition of drug efflux pumps and in the oncogenic signaling pathways.

In conclusion, LY294002 sensitized BCRP-overexpessing drug resistant cancer cells without affecting BCRP translocation. Due to its multifunctional effects, LY294002 can be used for developing more effective and tolerable reagents for cancer treatment.

\section{References}

1. Bates SE, Robey R, Miyake K, Rao K, Ross DD and Litman T: The role of half-transporters in multidrug resistance. J Bioenerg Biomembr 33: 503-511, 2001

2. Stavrovskaya AA: Cellular mechanisms of multidrug resistance of tumor cells. Biochemistry 65: 95-106, 2000.

3. Gottesman MM, Fojo T and Bates SE: Multidrug resistance in cancer: role of ATP-dependent transporters. Nat Rev Cancer 2: $48-58,2002$.

4. Ng SSW, Tsao MS, Chow S and Hedley DW: Inhibition of phosphatidylinositide 3-kinase enhances gemcitabine-induced apoptosis in human pancreatic cancer cells. Cancer Res 60: 5451-5455, 2000.

5. Ding S, Chamberlain M, McLaren A, Goh L, Duncan I and Wolf CR: Cross-talk between signalling pathways and the multidrug resistant protein MDR-1. Br J Cancer 85: 1175-1184, 2001.

6. Hu L, Hofmann J, Lu Y, Mills GB and Jaffe RB: Inhibition of phosphatidylinositol 3'-kinase increases efficacy of paclitaxel in in vitro and in vivo ovarian cancer models. Cancer Res 62 1087-1092, 2002.

7. Clark AS, West K, Streicher S and Dennis PA: Constitutive and inducible Akt activity promotes resistance to chemotherapy, trastuzumab, or tamoxifen in breast cancer cells. Mol Cancer Ther 1: 707-717, 2002.

8. Jin W, Wu L, Liang K, Liu B, Lu Y and Fan Z: Roles of the PI-3K and MEK pathways in Ras-mediated chemoresistance in breast cancer cells. Br J Cancer 89: 185-191, 2003.

9. Allikmets R, Schriml L, Hutchinson A, Romano-Spica V and Dean M: A human placenta-specific ATP-binding cassette gene (ABCP) on chromosome 4q22 that is involved in multidrug resistance. Cancer Res 58: 5337-5339, 1998.

10. Doyle LA, Yang W, Abruzzo LV, Krogmann T, Gao Y, Rishi AK and Ross DD: A multidrug resistance transporter from human MCF-7 breast cancer cells. Proc Natl Acad Sci USA 95: 15665-15670, 1998.

11. Miyake K, Mickley L, Litman T, et al: Molecular cloning of cDNAs which are highly overexpressed in mitoxantrone-resistant cells: demonstration of homology to $\mathrm{ABC}$ transport genes. Cancer Res 59: 8-13, 1999.

12. Jonker JW, Buitelaar M, Wagenaar E, et al: The breast cancer resistance protein protects against a major chlorophyll-derived dietary phototoxin and protoporphyria. Proc Natl Acad Sci USA 99: 15649-15654, 2002.

13. van Herwaarden AE, Jonker JW, Wagenaar E, Brinkhuis RF, Schellens JH, Beijnen JH and Schinkel AH: The breast cancer resistance protein (Bcrp1/Abcg2) restricts exposure to the dietary carcinogen 2-amino-1-methyl-6-phenylimidazo[4,5-b]pyridine. Cancer Res 63: 6447-6452, 2003.

14. Imai $Y$, Asada S, Tsukahara S, Ishikawa E, Tsuruo $T$ and Sugimoto Y: Breast cancer resistance protein exports sulfated estrogens but not free estrogens. Mol Pharmacol 64: 610-618, 2003 .
15. Suzuki M, Suzuki H, Sugimoto $Y$ and Sugiyama Y: ABCG2 transports sulfated conjugates of steroids and xenobiotics. J Biol Chem 278: 22644-22649, 2003.

16. Zhou S, Schuetz JD, Bunting KD, et al: The ABC transporter $\mathrm{Bcrp1/ABCG} 2$ is expressed in a wide variety of stem cells and is a molecular determinant of the side-population phenotype. Nat Med 7: 1028-1034, 2001.

17. Maliepaard M, van Gastelen MA, de Jong LA, et al: Overexpression of the BCRP/MXR/ABCP gene in a topotecanselected ovarian tumor cell line. Cancer Res 59: 4559-4563, 1999.

18. Kawabata S, Oka M, Shiozawa K, et al: Breast cancer resistance protein directly confers SN-38 resistance of lung cancer cells. Biochem Biophys Res Commun 280: 1216-1223, 2001.

19. Yanase K, Tsukahara S, Asada S, Ishikawa E, Imai Y and Sugimoto Y: Gefitinib reverses breast cancer resistance proteinmediated drug resistance. Mol Cancer Ther 3: 1119-1125, 2004.

20. Mogi M, Yang J, Lambert JF, et al: Akt signaling regulates side population cell phenotype via Bcrp translocation. J Biol Chem 278: 39068-39075, 2003.

21. Takada T, Suzuki H, Gotoh Y and Sugiyama Y: Regulation of the cell surface expression of human BCRP/ABCG2 by the phosphorylation state of Akt in polarized cells. Drug Meta Dispos 33: 905-909, 2005.

22. Bleau AM, Hambardzumyan D, Ozawa T, Fomchenko EI, Huse JT, Brennan CW and Holland EC: PTEN/PI3K/Akt pathway regulates the side population phenotype and ABCG2 activity in glioma tumor stem-like cells. Cell Stem Cell 4: 226-235, 2009.

23. Imai $Y$, Ishikawa $E$, Asada $S$ and Sugimoto $Y$ : Estrogen-mediated post transcriptional down-regulation of beast cancer resistant protein/ABCG2. Cancer Res 65: 596-604, 2005.

24. Kono T, Imai Y, Yasuda S, et al: The CD155/poliovirus receptor enhances the proliferation of ras-mutated cells. Int J Cancer 122: 317-324, 2008.

25. Imai $Y$, Tsukahara $S$, Ishikawa $E$, Tsuruo $T$ and Sugimoto $Y$ : Estrone and $17 \beta$-estradiol reverse breast cancer resistance protein-mediated multidrug resistance. Jpn J Cancer Res 93: 231-235, 2002.

26. Armstrong L, Hughes O, Yung S, et al: The role of PI3K/AKT, MAPK/ERK and NFkappabeta signalling in the maintenance of human embryonic stem cell pluripotency and viability highlighted by transcriptional profiling and functional analysis. Hum Mol Genet 15: 1894-1913, 2006.

27. Wieman HL, Wofford JA and Rathmell JC: Cytokine stimulation promotes glucose uptake via phosphatidylinositol-3 kinase/Akt regulation of Glut 1 activity and trafficking. Mol Biol Cell 18: 1437-1446, 2007.

28. Edinger AL and Thompson CB: Akt maintains cell size and survival by increasing mTOR-dependent nutrient uptake. Mol Biol Cell 13: 2276-2288, 2002.

29. Imai Y, Ohmori K, Yasuda S, Wada M, Suzuki T, Fukuda K and Ueda Y: Breast cancer resistance protein/ABCG2 is differentially regulated downstream of extracellular signal-regulated kinase. Cancer Sci 100: 1118-1127, 2009.

30. Srivastava AK: Inhibition of phosphorylase kinase, and tyrosine protein kinase activities by quercetin. Biochem Biophys Res Commun 131: 1-5, 1985.

31. Agullo G, Gamet-Payrastre L, Manenti S, Viala C, Rémésy C, Chap $\mathrm{H}$ and Payrastre B: Relationship between flavonoid structure and inhibition of phosphatidylinositol 3-kinase: a comparison with tyrosine kinase and protein kinase $\mathrm{C}$ inhibition. Biochem Pharmacol 53: 1649-1657, 1997.

32. Imai Y, Tsukahara S, Asada S and Sugimoto Y: Phytoestrogens/ flavonoids reverse breast cancer resistance protein/ABCG2mediated multidrug resistance. Cancer Res 64: 4346-4352, 2004.

33. Abdul-Ghani R, Serra V, Györffy B, Jürchott K, Solf A and Schäfer R: The PI3K inhibitor LY294002 blocks drug export from resistant colon carcinoma cells overexpressing MRP1. Oncogene 25: 1743-1752, 2006. 